\title{
Communication et numérique : entre métiers émergents et discours circulants
}

Le cas du secteur des télécommunications

Errecart Amaia

Maître de conférences à l'Université Paris 13 - Sorbonne Paris Cité Laboratoire des sciences de l'information et de la communication (LabSIC)

amaia.errecart@free.fr 


\section{Résumé}

$\mathrm{Au}$ carrefour du technique et du social, le numérique, s'appuyant sur le modèle d'Internet, a transformé les pratiques professionnelles, faisant apparaître de nouveaux métiers, de nouvelles configurations organisationnelles, de nouveaux usages, ainsi que de nouveaux discours. La technologie numérique y est présentée comme véritable vecteur de changement socio-culturel, dont l'entreprise entend faire de ses communicateurs des hérauts, des prophètes. Nous nous intéressons ici à un nouveau métier de l'Internet, dont la dénomination même porte la marque du registre religieux. "Évangéliste» est devenu une nouvelle fonction dans les entreprises de la sphère des télécommunications. La récurrence du terme participe de la construction d'une véritable rhétorique messianique autour du numérique.

Nous interrogeons l'apparition de cette nouvelle fonction de communicateur dans ce secteur, les enjeux associés à sa professionnalisation, et la construction d'une identité professionnelle attachée à ce nouveau métier à travers les logiques d'ethos auxquelles il donne lieu. À travers son étude, nous cherchons à analyser la prégnance du registre religieux qui se fait jour autour du numérique et ainsi interroger les imaginaires et les conceptions de la communication qu'il sous-tend.

Mots-clés: Numérique, télécommunications, analyse de discours, récit, ethos, imaginaire religieux, expertise, professionnalisation

\section{Abstract}

At the junction of the technical and social, digital technology, drawing on the model of the Internet, has transformed professional practices, by revealing new professions, new organizational configurations, new uses and new discourses. Digital technology is presented as a socio-cultural change vector, that companies ask their communicators to be prophets. We are interested in one of the new jobs of the Internet, whose name bears the mark of religious register: "evangelist" has become a new function in the sphere of business telecommunications and digital. The recurrence of the term involved the construction of a very messianic rhetoric around digital.

We analyse the merge of this new communicator function in this sector, the stakes associated with its professionalization and the construction of a professional identity attached to this new business through its logic of ethos. Through the study of the development of this new business speech communication, we seek to analyze the meaning of the religious register that is emerging around the digital and thus question the imaginary conceptions of communication that it subtends.

Keywords: Digital, telecommunications, discourse analysis, narrative approach, ethos, religious imagination, expertise, professionalization 
Au carrefour du technique et du social, le numérique, s'appuyant sur le modèle d'Internet, a transformé voire bouleversé les pratiques professionnelles. Sa généralisation a notamment fait apparaître de nouvelles configurations organisationnelles, de nouveaux usages, de nouveaux métiers. Elle a également fait émerger de nouveaux discours dans les organisations, dans lesquels la technologie numérique est présentée comme un véritable vecteur de changement socio-culturel, dont l'entreprise entend faire de ses communicateurs des hérauts, voire des prophètes. Milad Doueihi évoque ainsi le terme de "conversion », " aux deux sens $\mathrm{du}$ terme, technique et religieux » pour désigner «la transition de la technologie analogique au numérique » (Doueihi, 2011, p. 23). Philippe Breton avait en outre déjà mis en évidence la "religiosité » qui caractérise une grande partie des discours tenus sur le développement d'Internet (Breton, 2000).

C'est tout particulièrement à l'un des nouveaux métiers de l'Internet, dont la dénomination même porte la marque de ce registre religieux, que nous avons choisi de nous intéresser ici. "Evangéliste » est ainsi devenu une nouvelle fonction dans les entreprises de la sphère des télécommunications et du numérique: Vint Cerf, considéré comme l'un des pères fondateurs d'Internet, est ainsi Chief Internet Evangelist de Google; Microsoft propose des postes de Developer Evangelist et Technical Evangelist ; SFR possède son Chief Cloud Evangelist... La récurrence du terme participe de la construction d'une véritable rhétorique messianique autour du numérique.

En nous appuyant sur un corpus constitué de textes issus des sites Internet de deux entreprises françaises de télécommunications, Orange et Bouygues Telecom ${ }^{13}$, nous voulons ici interroger l'apparition de cette nouvelle fonction de communicateur dans ce secteur, les enjeux associés à sa professionnalisation, et la construction d'une identité professionnelle attachée à ce nouveau métier à travers les logiques d'ethos auxquelles il donne lieu. Partant du postulat que la «culture numérique» et son environnement en évolution permanente sont à « examiner comme un ensemble de pratiques discursives, qui ont leurs propres normes et conventions » (Doueihi, 2011, p. 26), nous nous appuierons sur la méthode que constitue l'analyse de discours (Bonnafous et Krieg-Planque, 2013). A travers l'étude de la mise en discours de ce nouveau métier de la communication, nous cherchons à analyser la prégnance du registre religieux qui se fait jour autour du numérique et ainsi interroger les imaginaires et les conceptions de la communication qu'il sous-tend.

\footnotetext{
${ }^{13}$ Précisons que ce corpus a été constitué au cours du mois de septembre 2014 ; il rassemble une centaine de textes datés de 2012, 2013 et 2014. Composé de captures d'écran issues des différentes rubriques des sites Internet, de rapports d'activité, d'interviews, il porte la marque - voulue - de l’hétérogénéité des supports de communication produits par les deux entreprises étudiées.
} 


\section{Technologie numérique et circulatin des discours}

« Appréhender le web en sciences humaines et sociales revient à vouloir saisir une réalité techniquement complexe et socialement construite, non pas seulement en ce qu'il donne à voir de pratiques qui peuvent émerger de l'observation sociosémiotique, mais également en ce qu'il instaure un ensemble de médiations de natures différentes (Monnoyer-Smith, 2013, p. 12). »C'est bien dans cette approche décrite par Laurence Monnoyer-Smith, s'inscrivant en droite ligne des travaux de Bruno Latour (Latour, 2010), que nous nous situons dans cette recherche, en mettant plus précisément la focale sur les médiations langagières et discursives.

Avant d'entrer dans l'analyse du nouveau métier du web qu'est celui d' " évangéliste » et des enjeux associés à sa professionnalisation, il nous faut commencer par décrire les contours de notre approche qui consiste à appréhender le numérique sous l'angle de la circulation des discours auxquels il donne lieu.

\subsection{L'entrée par l'analyse des médiations langagières}

Nous considérons Internet et les médias numériques plus généralement comme des dispositifs sociotechniques, dans la mesure où «l'imbrication du social et de la technique apparaît [...] nette dans les dispositifs du Web social où il paraît difficile d'isoler totalement ce qui relèverait d'un "pur technique" de l'univers d'un "pur social" (Proulx et Millerand, 2010, p. 17). " Or, nous choisissons de ne pas privilégier une approche par les médiations sociotechniques en tant que telles, qui consisterait à observer comment celles-ci sont «matérialisées, institutionnalisées, instrumentalisées, sémiotisées et comment elles construisent des formes d'exercice du pouvoir entre acteurs » (Monnoyer-Smith, 2013, p. 14), autrement dit comment ces médiations s'expriment dans des écrits d'écran et se traduisent par autant d'《 inscriptions supportées par un code numérique » (Bonaccorsi, 2013, p. 125).

Définissant la communication comme activité discursive (Krieg-Planque, 2012), nous choisissons de nous référer plutôt à l'analyse de discours dont l'éclairage nous paraît tout à fait pertinent ici, en vertu de «l'ambition critique qui l'anime depuis ses débuts » et parce qu'elle vise à «rendre compte à la fois des mutations de la communication et de la permanence d'enjeux symboliques » (Krieg-Planque et Oger, 2010, p. 95). Précisément, l'analyse de discours s'intéresse « aux formes et aux modalités d'expression des messages médiatiques, politiques, publics, organisationnels, etc. en rapport avec des cadres sociaux (le contexte historique, le média, le parti politique, le gouvernement, l'entreprise, etc.). Il s'agit d'une démarche fondée sur la linguistique, mais qui insiste sur le lien entre le discours et le social, entre le verbal et l'institutionnel, entre les mots, les figures, les arguments et ceux qui les énoncent et les interprètent » (Bonnafous et Krieg-Planque, 2013, p. 223). Ainsi, l'analyse de discours, relevant de l'approche pragmatique, envisage les 
énoncés à travers les conditions d'émergence de significations dans leur contexte d'apparition. Elle constitue une approche «qui se confronte nécessairement à la façon dont le locuteur, dans son discours, construit une identité, se positionne dans l'espace social et cherche à agir sur l'autre » (Amossy, 2010, p. 9). Les discours sont donc de véritables faits de communication, des objets de transaction symbolique entre acteurs. L'analyse va alors consister à révéler des éléments de compréhension non immédiatement perceptibles, à mettre au jour un certain «ordre du discours ", dans la mesure où « ce dont il s'agit ici, ce n'est pas de neutraliser le discours, d'en faire le signe d'autre chose et d'en traverser l'épaisseur pour rejoindre ce qui demeure silencieusement en deçà de lui, c'est au contraire de le maintenir dans sa consistance, de le faire surgir dans la complexité qui lui est propre " (Foucault, 1969, p. 65).

Or, l'analyse de discours, qu'elle prenne pour objet des institutions ou des entreprises, comme dans les deux cas que nous allons étudier, porte un intérêt spécifique à la question de la légitimation de ces organisations (Krieg-Planque et Oger, 2010, p. 91). Ainsi, soumises à cet impératif de légitimation et soucieuses de ménager l'adhésion à leur projet, tant en externe qu'en interne, elles recourent, selon Alice Krieg-Planque et Claire Oger, à deux principales ressources, caractéristiques des «discours autorisés 》: "la stabilisation des énoncés et l'effacement de la conflictualité » (Krieg-Planque et Oger, 2010, p. 94).

C'est bien dans cette perspective que nous inscrivons notre étude de terrain portant sur les entreprises de télécommunications Orange France et Bouygues Telecom. Précisons tout d'abord que ces entreprises constituent deux des quatre grands opérateurs dits de réseau mobile au sein du secteur des télécommunications français. Orange France, qui a succédé à France Télécom, ancien opérateur historique français, fait partie du groupe international de télécommunications Orange apparu au Royaume Uni en 1994. Le Groupe Orange constitue le premier opérateur en France sur le téléphone mobile, le fixe et Internet haut débit et le $4{ }^{\text {ème }}$ plus grand opérateur mondial, est implanté dans 30 pays en tant qu'opérateur grand public et compte 236 millions de clients et 165000 collaborateurs en 2013. Orange France constitue le pilier central de cette structure avec plus de 80000 salariés et $49 \%$ du chiffre d'affaires du groupe réalisé sur son territoire en $2013^{14}$. L'entreprise Bouygues Telecom, quant à elle, créée en 1994, se présente elle-même comme « un opérateur global de communications électroniques : mobile, fixe, télévision, Internet et cloud » comptant 9659 collaborateurs, 11,1 millions de clients mobile et 2 millions de clients haut débit fixe ${ }^{15}$. Sa sphère d'activité est essentiellement française.

\footnotetext{
${ }^{14}$ Sources : site institutionnel d'Orange France : http://orange.com/sirius/histoire/fr/histoire/

${ }^{15}$ Sources : site institutionnel de Bouygues Telecom :

$\mathrm{http}: / / \mathrm{www} . c o r p o r a t e . b o u y g u e s t e l e c o m . f r / n o u s-c o n n a i t r e /$ notre-entreprise
} 
L'entreprise est le troisième des quatre opérateurs de téléphonie mobile après Orange et SFR - le quatrième étant l'opérateur Free apparu en 2012.

C'est précisément leur position de, respectivement, numéro un et trois qui a motivé le choix de ces deux groupes : nous avons voulu nous intéresser d'une part au groupe leader de son secteur au sein du marché français et appartenant à un groupe international, Orange France; d'autre part, à un groupe français de dimension nettement inférieure, mais occupant une place structurante au sein des télécommunications de par son ancienneté - qui la distingue de Free, Bouygues Telecom.

Pour ces entreprises, concurrentes bien que de taille et d'envergure différentes, le développement des connaissances digitales, d'une « culture digitale », représente un enjeu stratégique crucial. Il constitue en effet un avantage concurrentiel qui « dépend de la détention de connaissances particulières ; il est alors logique qu'au sein d'un marché concurrentiel, les organisations concurrentes cherchent à s'approprier cette connaissance » (Ferrary et Pesqueux, 2011, p. 197).

Les discours produits par ces organisations vont ainsi se caractériser à la fois par une double dimension rhétorique et performative, et par le recours à des formes narratives et symboliques renvoyant à des imaginaires et des conceptions de la communication qu'il convient d'interroger.

\subsection{Des formes narratives à l'œuvre}

Ainsi, les «discours autorisés » que nous nous proposons d'analyser ont pour caractéristique de pouvoir être appréhendés en tant que « récits ». En effet, la notion de récit, empruntée à la narratologie, permet de «comprendre les productions symboliques et langagières visant l'unification, la structuration et le fonctionnement de groupes constitués autour d'un objectif de production de biens et de services » (D'Almeida, 2001, p. 9). A ce titre, l'approche narrative peut permettre une forme d'herméneutique de l'action collective organisée. Les discours organisationnels sont ici des récits au sens où ils possèdent une structure narrative - que l'analyse va permettre de mettre au jour, cherchent à proposer un sens - le terme étant pris dans sa polysémie, soit à la fois signification et direction, et ont une visée explicative et unificatrice. Ces «récits économiques» constituent donc « un ensemble de narrations qui proposent un sens, une signification et un but dans lesquelles l'entreprise se raconte en racontant le monde » (D'Almeida, 2004, p. 26).

C'est donc à cette aune que nous appréhendons les discours des groupes Orange et Bouygues Telecom portant sur l'émergence et la généralisation de la technologie numérique. Nous observons ainsi qu'un récit se fait jour et est orchestré autour de ce thème - crucial et éminemment stratégique pour les deux opérateurs globaux de communications électroniques. 
Parmi les caractéristiques inhérentes à tout récit, Jean-Michel Adam met la focale sur les points suivants : la succession d'événements, la notion de processus et la transformation des prédicats dans le temps (Adam, 2001) ${ }^{16}$. Selon cette approche, "raconter, c'est construire une intrigue, c'est-à-dire mettre dans un certain ordre textuel (racontant) la suite des événements et des actions qui constitue l'histoire racontée » (Adam, in : Charaudeau et Maingueneau, 2002, p. 486).

Ainsi, à la rubrique « Nous connaître » du site Internet de Bouygues Telecom, puis « Notre histoire», on peut découvrir une frise chronologique qui décrit, année par année, ce qui est présenté par l'entreprise comme «l'histoire de Bouygues Telecom de 1996 à nos jours : 16 ans d'innovations et d'événements numériques ${ }^{17}$. L'entreprise s'y met en scène en empruntant au registre épique : recourant au « nous » organisationnel, inclusif et fédérateur, elle énumère les actions, réalisations, obstacles franchis, défis relevés au fil des années, qui apparaissent comme autant de hauts faits victorieux.

Le groupe Orange entend également inscrire son histoire dans le temps long cherchant à construire une représentation mémorielle de l'entreprise - et dans le registre épique. Il choisit de remonter aux origines des télécommunications, faisant coïncider histoire de l'émergence de ces technologies et histoire du groupe : « 200 ans de communications et d'innovations au service de 230 millions de clients » peuton lire à la rubrique « Histoire » du site institutionnel, qui donne le choix entre deux entrées : «Les moments forts » et «Toute 1'histoire » qui prend là encore la forme d'une frise chronologique illustrée ${ }^{18}$. Le groupe insiste sur l'ancrage dans la durée, le temps long, la continuité, en se présentant comme "héritier d'une histoire plurielle », « fier de ses racines », et en évoquant la « mémoire de l'entreprise, d'hier à aujourd'hui ». Le recours à ce registre temporel et historique s'accompagne, comme chez Bouygues Telecom, du registre épique, qui transparaît à travers les expressions " défis relevés », " innovations audacieuses », " conquêtes à l'échelle du monde », « épopée à découvrir » 19 .

On le voit, les deux entreprises se mettent en récit et leur communication «peut être comprise comme l'interminable récitation de succès réels ou à venir » (D’Almeida, 2001, p. 91). Construisant une représentation héroïsée d'elles-mêmes, elles entendent produire une forme de légende à partir de leur propre histoire (au sens étymologique de legenda : ce qui doit être lu et dit).

\footnotetext{
${ }^{16}$ Selon Jean-Michel Adam, six constituants doivent être réunis pour que l'on puisse parler de récit : une succession d'événements, une unité thématique, des prédicats transformés, un procès, la causalité narrative d'une mise en intrigue et l'évaluation finale (explicite ou implicite) (Adam, 2001, p, 46 à 56).

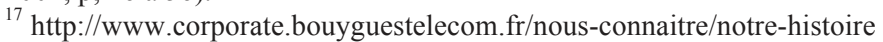

${ }^{18} \mathrm{http}: / /$ orange.com/sirius/histoire/fr/accueil

${ }^{191}$ bid.
} 
Or, cette «légende» s'appuie sur deux thèmes récurrents dans les discours étudiés : le thème du pionnier et celui de l'innovation, qui se trouvent fréquemment en situation de cooccurrence, comme dans les verbatim suivants, d'abord dans les discours de Bouygues Telecom :

L'entreprise s'est toujours démarquée par ses offres innovantes $(\ldots)^{20}$.

Inventeur du premier forfait Mobile en 1996, Bouygues Telecom lance les premières offres illimitées avec Millenium en 1999 et Neo en 2006. En 2007, Bouygues Telecom propose la première offre de convergence fixe-mobile pour les entreprises. [...] Le 18 juin 2012, Bouygues Telecom a lancé la Bbox Sensation, une nouvelle gamme de Box qui intègre les technologies les plus innovantes au service du foyer numérique ${ }^{21}$.

Puis, dans ceux d'Orange France :

Notre groupe bénéficie de 40 ans d'expérience dans l'amélioration des techniques de communication grâce à des infrastructures haut de gamme et des services innovants qui ont marqué l'histoire des télécommunications, depuis les premiers services télématiques élaborés dans les années 1980 en France, jusqu'aux solutions les plus innovantes offertes par les plateformes NFC, le Cloud, la 4G, la fibre optique et les services IPTV que nous lançons aujourd'hui au niveau mondial ${ }^{22}$.

L'innovation est le moteur d'Orange. [...] Nous bâtissons le premier opérateur de l'ère Internet, avec de nouveaux modèles générateurs de valeur pour l'ensemble de nos parties prenantes ${ }^{23}$.

A travers la conjugaison de ces deux thèmes, c'est un récit de la conquête (des clients, des marchés), de la réussite technologique et économique qui se décline et s'affirme. On voit ainsi que «la narration, comme récit et instrument de transformation, est utilisée, le plus souvent, pour mobiliser les énergies internes et les ressources externes [...]. La narration y est janusienne : tournée vers le passé, elle rapporte avec nostalgie les succès anciens ; orientée vers le futur, elle appelle à une grande aventure. Dans les deux cas, elle se veut une épopée relatant, après une lutte difficile contre la compétition, la performance glorieuse du stratège et de ses troupes » (Giroux et Marroquin, 2005, p. 23).

\footnotetext{
${ }^{20} \mathrm{http} / / / \mathrm{www}$. corporate.bouyguestelecom.fr/nous-connaitre/notre-entreprise

${ }^{21}$ Ibid.

${ }^{22} \mathrm{http} / / /$ www.orange.com/fr/a-propos/Groupe/notre-marque

${ }^{23} \mathrm{http} / / /$ www.orange.com/fr/innovation/l-innovation-selon-Orange/moteur-de-la-revolutionnumerique
} 
Accompagnant ce récit de la conquête victorieuse qui s'appuie sur les thèmes du pionnier et de l'innovation permanente, apparaît une autre forme de narration dans les discours analysés que l'on pourrait qualifier de «récit de l'émancipation ». En effet, les deux entreprises se posent en pédagogues des technologies numériques, en passeurs, en facilitateurs auprès de tous leurs publics, comme l'indique l'expression récurrente de «libérer les usages» dans les discours de Bouygues Telecom :

En tant qu'opérateur global, Bouygues Telecom souhaite répondre aux besoins variés de ses clients grâce à un éventail complet d'offres et de services, tant mobiles que fixes, tout en libérant toujours plus les usages ${ }^{24}$.

En 2012, Bouygues Telecom a continué de libérer l'usage de ses clients ayant un accès Internet mobile ${ }^{25}$.

De même, on peut noter la récurrence des verbes «faciliter » et «simplifier » dans ceux d'Orange France :

Nous ne cherchons pas seulement à investir dans les réseaux très performants, mais nous touchons également les produits et services qui permettent de faciliter la vie de nos plus de 239 millions de clients ${ }^{26}$.

Notre slogan « La vie change avec Orange » incarne cet engagement quotidien de faciliter pour tous le passage au numérique ${ }^{27}$.

Orange innove pour simplifier l'accès au monde digital. [...] La digitalisation du monde s'accélère et l'autonomie aussi ${ }^{28}$.

Comme l'indique ce dernier verbatim, l'idée d'une entreprise qui entend faciliter, libérer, ouvrir des perspectives - technologiques et au-delà - à ses clients, se retrouve avec les thèmes de l'autonomie et de l'émancipation à l'égard des contraintes techniques liées à l'outil numérique. On peut ainsi lire sur le site de Bouygues Telecom :

Site d'information très complet, bouyguestelecom.fr tend à offrir toujours plus d'autonomie aux internautes ${ }^{29}$.

De l'indépendant au grand compte, Bouygues Telecom Entreprises propose aux différents types de sociétés des offres dédiées et un accompagnement sur mesure, pour communiquer sans contrainte, de manière efficace et fiable ${ }^{30}$.

\footnotetext{
${ }^{24}$ Bouygues Telecom, L'essentiel 2012, p. 12.

${ }^{25}$ Ibid., p. 13.

${ }^{26} \mathrm{http}: / /$ www.orange.com/fr/a-propos/Groupe/notre-marque

${ }^{27}$ Ibid.

${ }^{28} \mathrm{http} / /$ bienvivreledigital.orange.fr/autonomie/orange-innove-pour-laccessibilite

${ }^{29}$ Bouygues Telecom, L'essentiel 2012, p. 21.

${ }^{30}$ Ibid., p. 18.
} 
Ou encore, sur le site d'Orange France, à la rubrique « Bien vivre le digital », dont l'intitulé même évoque la recherche du confort d'accès, du bien-être et d'un certain hédonisme :

Le monde digital pour tous, c'est un accès libre et facilité aux nouveaux médias [...]. Des solutions innovantes existent pour rendre la vie plus facile $e^{31}$.

Se familiariser avec Internet, ce n'est plus un casse-tête avec Internet Facile d'Orange! Avec l'option Internet facile, faites vos premiers pas sur le web en toute sérénité, même si vous n'avez jamais touché d'ordinateur ! ${ }^{32}$.

En somme, semblant vouloir aller au-delà du service dû au client, les deux entreprises de télécommunications disent vouloir offrir à ce dernier liberté, autonomie, fluidité, absence de contraintes, simplicité et aisance dans son rapport à la technologie numérique. Facilitant sa maîtrise des outils, elles lui ouvriraient des possibles, des horizons communicationnels et personnels, gage pour l'individu de libération, de réalisation de soi et d'épanouissement.

A cette dimension de l'émancipation, s'ajoute la référence fréquente à la « communauté », et plus particulièrement à l' " entraide communautaire », comme dans les extraits suivants issus du site de Bouygues Telecom :

En juillet 2011, avec B\&YOU, Bouygues Telecom lançait une offre mobile inédite sans engagement et à petit prix [...]. La marque $100 \%$ en ligne innovait avec un concept basé sur l'entraide communautaire. En 2012, B\&YOU est demeurée à l'écoute de sa communauté et a régulièrement amélioré ses offres et services ${ }^{33}$.

Communautaire et participatif, B\&YOU est constamment à l'écoute des suggestions d'amélioration proposées par sa communauté d'internautes. Cette communauté s'entraide sur le site b-and-you. $\mathrm{fr}^{34}$.

L'entreprise, devenue organisation communautaire, semble vouloir faire oublier ici son ethos économique et sa finalité lucrative; elle se présente sur un mode axiologique, valorisant les pratiques de coopération et de solidarité en son sein, ainsi que la constitution de réseaux de sociabilité, activés par les réseaux informatisés.

De même, le site institutionnel d'Orange France proclame à la rubrique « Notre marque »: " Nous formons une communauté internationale de 164000 personnes $[\ldots] »^{35}$. La rubrique «Bien vivre le digital », déjà évoquée, possède un onglet

\footnotetext{
${ }^{31} \mathrm{http} / / /$ bienvivreledigital.orange.fr/autonomie

${ }^{32} \mathrm{http}$ ///bienvivreledigital.orange.fr/autonomie/chacun-sa-solutio

${ }^{33}$ Bouygues Telecom, L'essentiel 2012, p. 16.

${ }^{34} \mathrm{http}: / / \mathrm{www} . c 0 r p o r a t e . b o u y g u e s t e l e c o m . f r / n o u s-c o n n a i t r e / n o t r e-e n t r e p r i s e$

$35 \mathrm{http}$ //www.orange.com/fr/a-propos/Groupe/notre-marque
} 
«Communauté Orange» qui indique «Rejoignez la communauté Orange » ${ }^{36}$, l'impératif sonnant comme une invite.

On voit ici que la communauté semble constituer une «entité fondée sur une interaction permanente entre individus partageant à la fois des biens et des centres d'intérêt communs. Dans ce schéma, la communauté s'interpose comme la médiation sociale essentielle entre l'individu et la société. Comme le principal lieu des échanges culturels et informationnels » (Rebillard, 2007, p. 21).

Les discours étudiés témoignent par conséquent de la construction d'un triple récit: récit de la conquête, de l'émancipation et de la communauté. Or, ces trois récits, qui n'en forment qu'un, renvoient en droite ligne aux origines du média Internet, à l'histoire de sa conception et à l'esprit de ses fondateurs (Cardon, 2010). En effet, les origines mêmes d'Internet nous montrent que celui-ci «n'a pas été conçu pour permettre à un émetteur de s'adresser à une masse de récepteurs, mais pour faciliter les échanges entre des individus tour à tour émetteurs et récepteurs. Les premiers protocoles qui ont donné naissance à la communication entre ordinateurs ont été pensés comme des outils d'échanges interpersonnels, non comme des médias. L'histoire d'Internet peut d'ailleurs se lire comme un élargissement illimité, passant d'une communication en face-à-face à une communication de plusieurs à plusieurs qui finit par s'étendre à tous » (Cardon, 2010, p. 8-9). L'élargissement ici évoqué serait donc in fine celui de l'espace public, et par conséquent de la démocratie elle-même et des valeurs d'autonomie, d'égalité et de transparence qui lui sont attachées.

Ainsi, le dispositif sociotechnique qu'est Internet s'est construit et développé en s'appuyant sur un ensemble de croyances et de valeurs faisant la part belle à l'innovation collaborative, à la liberté créative, à l'invention de « formes inédites de partage du savoir, de mobilisation collective et de critique sociale » (Cardon, 2010, p. 8). On le voit, les formes de narration produites par les entreprises Orange et Bouygues Telecom renvoient directement à ces croyances et valeurs constitutives du " grand récit» fondateur du média Internet.

\section{Entre identité discursive et identité professionnelle : émergence d'une nouvelle fonction de communicateur}

Emerge dans les discours constituant notre corpus la référence à un nouveau métier de la communication dont l'apparition est concomitante de la généralisation du numérique: celui d' «évangéliste». Cette nouvelle fonction va porter, personnifier le triple récit que nous venons de décrire. Afin d'interroger les enjeux

\footnotetext{
${ }^{36} \mathrm{http}: / /$ communaute.orange.fr/
} 
communicationnels et symboliques associés à sa professionnalisation, nous allons commencer par nous intéresser à la manière dont cette nouvelle fonction se met en discours, à travers notamment l'exploration des logiques d'ethos. Nous allons, pour cela, croiser les discours portant spécifiquement sur l'ethos de 1' " évangéliste », et des discours relevant plus largement du discours sur soi des deux organisations et donc d'une forme d'ethos institutionnel. Précisons que notre approche consiste à conjuguer les notions de "présentation de soi », empruntée à la sociologie, et d' ethos, ou image de soi que l'orateur produit dans son discours, empruntée à la rhétorique et à l'analyse du discours, afin d'appréhender la mise en scène de soi « dans ses multiples manifestations au sein d'une approche unifiée qui met l'accent à la fois sur la construction discursive de l'identité et sur l'efficacité verbale" (Amossy, 2010, p. 6). Nous concevons donc l'ethos comme une « dimension intégrante du discours »(Amossy, 2010, p. 7). Nous proposerons ensuite une réflexion sur les implications induites en termes d'imaginaires et de conceptions de la communication.

\subsection{Imaginaire religieux et logiques d'ethos}

«Evangéliste» semble être devenu une nouvelle profession née de l'essor d'Internet et de la technologie numérique, au vu des discours des deux entreprises de télécommunications que nous étudions. Ce constat est confirmé par une recherche portant sur d'autres organisations de ce secteur sur le moteur de recherche Google ; notons la forte prégnance du terme - qui apparaît presque systématiquement sous sa forme anglaise : evangelist - en contexte anglo-saxon. L'origine du terme de technology evangelist est associée à l'entreprise américaine Apple : c'est à Guy Kawasaki, l'un des premiers responsables marketing d'Apple dans les années 1980, que l'on doit le concept d'evangelism appliqué aux nouvelles technologies; et Mike Boich, promoteur de l'ordinateur Macintosh d'Apple Inc., est considéré comme le premier évangéliste technologique. Les dimensions technologique, communicationnelle et marketing semblent donc, dès l'origine de la fonction, intrinsèquement liées. Notons qu'en novembre 2006 apparaît aux Etats-Unis une première structuration professionnelle de ce nouveau métier : l'association professionnelle Global Network of Technology Evangelists est fondée par des évangélistes issus de Microsoft, Sun Microsystems et Yahoo !.

Si l'on considère les discours produits par le groupe Orange, à la rubrique «Orange Partner» - dédiée aux professionnels, il est question d'un $A P I^{37}$ Evangelist, d'un Orange Partner Evangelist ${ }^{38}$, d'un API Developer Evangelist ${ }^{39}$.

\footnotetext{
${ }^{37}$ L'expression anglo-saxonne API signifie « Application Programming Interface ».

${ }^{38} \mathrm{https}$ //www.orangepartner.com/articles/api-strategy-conference
} 
60 Le communicateur bousculé par le numérique

Quant au site de Bouygues Telecom, il fait notamment référence à des fonctions de Big data Evangelist et de Open API Evangelist.

Il est intéressant de souligner que la référence à ce métier émergent s'accompagne d'un usage récurrent du verbe "évangéliser ». Au sein d'une rubrique du site d'Orange consacrée à «Orange et le transmédia », on peut par exemple lire parmi les fonctions assignées au «transmédia Lab»: «évangéliser, informer sur les différents projets internationaux, mêlant à la fois nouveaux écrans, interactions, nouvelles technologies de manière ludique ${ }^{40}$.

L'expression " évangéliser une culture numérique » apparaît ainsi à la fois comme un leitmotiv et comme une injonction, dans les supports de communication interne de l'entreprise Bouygues Telecom (Mila, 2013). Notons qu'elle n'est pas absente de ses supports de communication externe; on en trouve notamment trace sur le site «Giiks », présenté par l'entreprise elle-même comme «le mag du geek par Bouygues Telecom » ou encore «le blog des nouvelles technologies ». On peut y lire les extraits suivants : «la société compte sur ses partenariats pour évangéliser ses futurs clients ${ }^{41}$; ou encore : «Microsoft continue à évangéliser auprès des développeurs, donc il n'y a aucun souci pour l'avenir $»^{42}$.

Cet usage du verbe «évangéliser» frappe par sa référence directe au registre religieux, et plus particulièrement chrétien. Si l'on considère son étymologie, le verbe « évangéliser » est issu du grec euaggélion qui signifie "bonne nouvelle » et peut être défini par " prêcher la bonne nouvelle (du Christ), annoncer l'Evangile », cette dernière renvoyant à «l'ensemble des livres contenant la doctrine du Christ» (Baumgartner et Ménard, 1996, p. 303). Dans la religion chrétienne, la pratique évangélisatrice renvoie ainsi à l'activité de diffusion et de propagation de la foi qui incombe à l'institution - comme à tout fidèle. En effet, la prédication, la « transmission de la parole » sont parmi les fonctions que l'Eglise s'assigne depuis son origine (Douyère, 2010a, p. 78). L'évangélisation, qui constituerait donc l'une des missions principales de l'Eglise, possède « un sens autant religieux et eschatologique que social, économique et politique. [...] Cette communication du kerygme $^{43}$ chrétien et cette incitation à la conversion sont pensées comme communication du "discours juste" qui doit occuper l'ensemble de l'espace social, et entretient en tant que tel des affinités avec la communication moderne d'entreprise, tant interne qu'externe » (Douyère, 2010b, p. 150).

\footnotetext{
${ }^{39} \mathrm{https}: / /$ www.orangepartner.com/content/past-events

${ }^{40} \mathrm{http} / / / \mathrm{www}$.orange.com/fr/a-propos/contenus/Orange-et-les-contenus/transmedia/Orange-et-letransmedia

${ }^{41} \mathrm{http} / / /$ www.giiks.com/smartphone/ctia-2008-protopage-1182/

${ }^{42} \mathrm{http} / / /$ www.giiks.com/smartphone/test-nokia-lumia-800-27040/

${ }^{43}$ Le kerygme désigne la diffusion du message chrétien, soit la «"proclamation" de la "bonne nouvelle" chrétienne : la venue du royaume de Dieu et le salut de l'humanité dans le Christ, Dieu fait homme » (Douyère, 2010b, p. 145).
} 
On songe ici à Milad Doueihi lorsqu'il compare la « culture numérique » en plein essor, avec sa tendance universaliste, à la religion, en évoquant le terme de conversion : «c'est précisément en raison de ce processus de conversion, apparemment inévitable, que la culture numérique [...] est en train de revendiquer un statut équivalent à celui d'une religion mondiale, avec ses prophètes et ses prêtres, ses institutions et ses chapelles, ses croyants, ses contestataires et ses schismatiques » (Doueihi, 2011, p. 23).

Si l'on se réfère à une analyse en termes d'ethos, on voit se dessiner autour de la figure de l'évangéliste - celui qui va donc, littéralement, porter et diffuser la bonne parole numérique - la construction d'un véritable ethos messianique - inscrit dans la dénomination même d' "évangéliste », de par son appartenance au registre religieux. Or, cet ethos s'intègre dans un «discours messianique d'ensemble prononçant l'avènement d'un nouvel Internet, fondé sur le social networking ${ }^{44}$, et décliné en partage de données, travail collaboratif, intelligence collective et démocratisation des médias » (Rebillard, 2007, p. 11).

Qu'il possède une connotation religieuse ou plus laïque, le messianisme est ainsi bien présent dans les discours étudiés, à l'image de ces extraits du site d'Orange France :

L'innovation est le moteur de la révolution numérique qui change nos vies. L'innovation est le moteur d'Orange ${ }^{45}$.

La vocation du Digital Society Forum est d'éclairer les changements sociétaux importants induits par le numérique. [...] Le Digital Society Forum est une preuve concrète de l'engagement du Groupe Orange, légitime par son ADN d'opérateur historique à éclairer les usages des nouvelles technologies ${ }^{46}$.

Sur le site de la Fondation Orange, le numérique est qualifié de «magnifique levier dans le domaine éducatif», d' "outil merveilleux pour aider les jeunes à retrouver le goût de l'école ${ }^{47}$. De même, l'évolution des usages liée au « transmédia » est qualifiée d' "expérience valorisante, attractive, communautaire, dans laquelle les créateurs deviennent véritablement acteurs de l'histoire... C'est l'avènement du transmédia ${ }^{48}$.

Présenté comme une véritable panacée, le numérique ouvrirait ainsi tous les possibles, en atteignant un «nouveau stade d'expansion sociétal » (Rebillard, 2007,

\footnotetext{
${ }^{44}$ Le social networking désigne la constitution de réseaux sociaux.

${ }^{45} \mathrm{http}$ //www.orange.com/fr/innovation/l-innovation-selon-Orange/moteur-de-la-revolutionnumerique

${ }^{46} \mathrm{http} / / /$ digital-society-forum.orange.com/fr/le-projet/7-1._a_propos_de_digital_society_forum

${ }^{47} \mathrm{http}: / / \mathrm{www}$.fondationorange.com/-solidarite-numerique-27-

${ }^{48} \mathrm{http} / / / \mathrm{www}$. orange.com/fr/a-propos/contenus/Orange-et-les-contenus/transmedia/Orange-et-letransmedia
} 
p. 11). L'évangéliste apparaît comme le "prophète " annonçant cet avènement, en même temps que le "prêtre » en interaction avec une communauté de "fidèles ", pour reprendre les termes de Milad Doueihi (Doueihi, 2011, p. 23).

Or, cet ethos messianique attaché à la figure de l'évangéliste s'accompagne d'une autre forme d'ethos, destiné à construire la légitimité professionnelle de cette nouvelle fonction, un ethos que nous qualifierons d' « expert». En effet, la fonction d'évangéliste est évoquée, dans les discours des deux entreprises que nous étudions, au sein des rubriques des sites institutionnels dédiées aux publics professionnels (et non au grand public). Elle est en outre associée presque systématiquement à l'idée de haute technologie, d'innovation de pointe, de créativité et d'excellence dans la compétence technique. C'est le cas notamment sur le site « Orange Partner» où le rôle d'un Orange Partner Evangelist se voit défini en anglais :

He can help you understand the value of our APIs for your service, while providing support for their integration ${ }^{49}$.

En témoigne également la rubrique du site d'Orange France consacrée au « réseau Orange $\mathrm{Fab}$ », où sont cités nommément plusieurs evangelists internes ou non au groupe - y figure notamment le Senior Creative Cloud Evangelist d'Adobe Systems. Ce réseau de professionnels destiné à des professionnels est ainsi décrit :

Après le succès de l'accélérateur de start-up Orange Fab dans la Silicon Valley, Orange développe un véritable réseau d'accélérateur dans le monde [...]. Orange associe son expérience de l'industrie des télécommunications à celle des mentors venus des écosystèmes technologiques locaux [...]. Les start-up bénéficieront d'un véritable réseau international ancré dans des écosystèmes innovants et dynamiques $[\ldots]^{50}$.

Notons que le terme de «mentor» est repris dans la même rubrique, associé au terme d' « expert», dans une adresse aux clients professionnels :

Nous sommes fiers d'avoir accès à un vivier des plus brillants experts et mentors au sein de notre écosystème. [...] Notre programme d'accélérateur exclusif permettra un échange exceptionnel avec nos experts : un conseil individualisé, en lien direct avec les mentors qui correspondent à vos besoins, à un retour des cadres supérieurs d'Orange sur les marchés potentiels que vous ciblez. Orange Fab est conçu pour vous assurer une expérience sur mesure avec chacun de nos experts $^{51}$.

\footnotetext{
${ }^{49} \mathrm{https}: / /$ www.orangepartner.com/articles/api-strategy-conference

${ }^{50} \mathrm{http}$ ://www.orangefabfrance.fr/a-propos/

${ }^{51} \mathrm{http}: / /$ www.orangefabfrance.fr/les-intervenants/
} 
L'association des termes d' «expert» et de «mentor» est intéressante à souligner : elle permet d'allier la caution de professionnalisme et de haut niveau de compétences apportée par l' « expert» à la dimension pédagogique et de conseil qu'évoque le terme de « mentor $»^{52}$.

Cet ethos expert va se traduire par un ton didactique lorsque l'entreprise, par la voix de ses «évangélistes" du numérique, dispense des conseils à ses clients professionnels, le public des entreprises. Le ton s'y fait même souvent prescriptif, comme dans ces verbatim issus de la rubrique «Comment animer son réseau social ? » du magazine en ligne de Bouygues Telecom dédié aux professionnels, le «Mag'Pro Bouygues Telecom » :

\footnotetext{
Une fois inscrit sur une plate-forme comme Viadeo, LinkedIn, Twitter ou Facebook, il faut désormais apprendre à faire vivre sa communauté $e^{53}$.

Votre présence sur les réseaux sociaux ne doit pas être uniquement motivée par une simple opportunité commerciale ${ }^{54}$.

L'intérêt d'un réseau social réside dans la communauté qu'on arrive (ou pas) à créer. [...] Une fois que l'on a ciblé une relation, il faut s'y intéresser, la solliciter sans la déranger et lui montrer de l'intérêt sans forcément attendre un retour direct. Il est impératif de maintenir le dialogue direct et indirect ${ }^{55}$.

Il ne faut pas hésiter à régulièrement rencontrer vos membres les plus influents / actifs $^{56}$.

Ne vous arrêtez pas de chercher de nouveaux contacts, car ce serait vous priver de possibles liens fructueux à l'avenir ${ }^{57}$.
}

On voit à travers la récurrence du déontique « il faut », ou « il est impératif de », comme l'utilisation du mode impératif (« ne vous arrêtez pas »), que l'entreprise, adoptant un discours prosélyte et dispensant la «bonne parole numérique », entend faire de ses clients eux-mêmes des relais de ce discours. Se dessine le double objectif de ces productions discursives : elles ont vocation à la fois à «éduquer» le client afin de le « convertir », et à créer un marché.

Nous voyons par conséquent que le recours à l'outil rhétorique que constitue l'ethos se traduit par la construction d'une image de soi favorable, susceptible de conférer autorité et crédibilité (Amossy, 2010, p. 5). Entre ethos messianique et ethos expert se fait jour ici la volonté de construire dans les discours la légitimité professionnelle d'un métier émergent de la communication. La fonction stratégique

\footnotetext{
${ }^{52}$ Rappelons que le terme « mentor » vient du nom d'un personnage de l'Odyssée, conseiller de Télémaque, le fils d'Ulysse, pris en français au sens de «sage conseiller» (Baumgartner \& Ménard, 1996, p. 492).

${ }^{53} \mathrm{http}: / /$ www.bouyguestelecom-pro.fr/lemag/web/comment-animer-son-reseau-social

${ }^{54}$ Ibid.

${ }^{55}$ Ibid.

${ }^{56}$ Ibid.

${ }^{57}$ Ibid.
} 
64 Le communicateur bousculé par le numérique

de l'évangéliste est mise en avant; il se voit «endosser l'habit de traducteur, vulgarisateur, simplificateur, ou encore de passeur, de médiateur » (De la Broise et Brulois, 2010, p. 129).

\subsection{Entre récit utopique et idéologie techniciste}

Nous nous situons donc ici au cœur de la conception performative du langage, telle qu'elle est mise en avant par la pragmatique et plus particulièrement la théorie des actes de langage ou speech acts (Austin, 1970), dans la mesure où celle-ci "instaure un lien, essentiel en argumentation, entre le langage et l'action» (Danblon, 2005, p. 107). En effet, selon la théorie austinienne, « "dire", c'est sans doute transmettre à autrui certaines informations sur l'objet dont on parle, mais c'est aussi "faire", c'est-à-dire tenter d'agir sur son interlocuteur, voire sur le monde environnant » (Kerbrat-Orecchioni, 2002, p. 16). La démarche pragmatique permet de comprendre comment le langage peut produire du sens et constituer un moyen d'influence. Elle suppose l'idée d'un pouvoir du verbe agissant et susceptible de faire agir, d'influencer, de construire des rapports sociaux, dans la mesure où « un discours est toujours et nécessairement un acte de parole» (Todorov, 1978, p. 48). La communication est donc ici conçue comme acte pour agir sur les représentations de ceux à qui elle s'adresse. Nous pouvons donc, selon cette approche, mettre au jour une « forme de l'échange » et intégrer dans la réflexion sur la communication la dimension sociale et symbolique de l'échange, sa dimension intentionnelle, « conative » selon Jakobson (Jakobson, 1963, p. 216).

Précisément, dans cette perspective, à travers la prégnance de la rhétorique messianique que nous avons pu mettre au jour, c'est bien tout un imaginaire de la communication qui est construit et diffusé. En effet, de la même façon que les travaux de Max Weber ont souligné que la dynamique du capitalisme n'était pas réductible à des facteurs objectifs mais que l'entrepreneuriat pouvait être mu par des fondements religieux, éthiques et psychologiques (Weber, 2000), « la compétence numérique véhicule un imaginaire social qui met en jeu non seulement la dimension virtuelle des technologies nouvelles, mais aussi certains postulats et préjugés religieux, historiques et politiques : ceux-ci façonnent la nouvelle culture avec autant de force et d'influence, voire plus, que le simple fait de l'accès à la même technologie par-delà les frontières nationales et culturelles » (Doueihi, 2011, p. 14).

Retraçant l'histoire des moyens de communication, Roger Bautier souligne ainsi « la prégnance de la discussion religieuse » et constate qu'il est « tout à fait possible de parler des moyens de communication en des termes quasi religieux » (Bautier, 1994, p. 35). Si le «culte de l'information » apparaît au sein de la cybernétique de Norbert Wiener, d'autres approches ont contribué à cette vision quasi religieuse de 
la communication - citons notamment l'influence du jésuite Teilhard de Chardin avec sa notion de "noosphère " ${ }^{58}$ ou de Marshall McLuhan avec son "village global ». Or, comme le souligne Franck Rebillard, « on aurait bien tort de négliger cette part d'imaginaire dans le déploiement des technologies, au prétexte du caractère délirant ou « irréaliste » de nombre de ces discours d'accompagnement. Si l'imaginaire constitue à n'en point douter la composante la plus immatérielle de la technique, elle n'en est pas moins partie prenante de son devenir social» (Rebillard, 2007, p. 83).

L'imaginaire que nous pouvons observer ici à partir de l'émergence du métier d'évangéliste possède deux caractéristiques majeures : il évoque un récit utopique du progrès - le progrès sociétal étant, selon cette vision, naturellement généré par le progrès technologique, et son corollaire : la croyance en une idéologie techniciste.

Citons pour exemple le verbatim suivant, issu de la rubrique « Notre marque » $d u$ site d'Orange France, qui fait explicitement référence à la notion de progrès :

Tout au long de cette période de progrès technologique qui a touché le monde entier, nous nous sommes attachés à aider nos clients à communiquer, interagir et collaborer davantage. Orange offre un soutien puissant et positif dans les périodes de changement ${ }^{59}$.

Ou encore cet extrait d'un discours du Président Directeur Général d'Orange, à la rubrique « Notre ambition », où transparaît l'idée d'un déterminisme technique :

La révolution numérique a tout changé dans nos vies. [...] J'ai pour ma part une conviction forte: celle que le numérique est un formidable outil de développement économique et social, un véritable catalyseur d'innovation, qui doit être accessible à tous et mis au service de tous ${ }^{60}$.

On voit dans ces propos se dessiner tout un univers de croyances dans la toutepuissance des nouveaux dispositifs techniques de communication61: « cette vision - au sens fort - est celle d'un monde idéal qui serait tout entier forme, comportement, information, message, communication, un monde fait d'éléments toujours en mouvement, en échange, en interaction. [...] Il s'agit d'une mystique de

\footnotetext{
${ }^{58}$ Pierre Teilhard de Chardin définissait la «noosphère» comme une «nappe d'énergie psychique » qui s'étend sur la Terre au fur et à mesure que l'homme en prend possession, et voyait le futur de l'humanité comme un nouveau « collectivisme» (Breton, 2000, p. 9).

${ }^{59} \mathrm{http} / / /$ www.orange.com/fr/a-propos/Groupe/notre-marque

${ }^{60} \mathrm{http} / / / \mathrm{www}$. orange.com/fr/engagements/responsabilite/vision

${ }^{61}$ Notons, dans cette perspective, que Guy Kawasaki cité précédemment est l'auteur d'un ouvrage paru aux Etats-Unis en 2011 dont le titre est éloquent : Enchantment, The Art of Changing Hearts, Minds and Actions. Il est traduit en français par L'art de l'enchantement.
} 
66 Le communicateur bousculé par le numérique

la forme et du message, qui mobilise les mêmes ressources affectives que celles qui se mettent au service des religions constituées » (Breton, 2000, p. 8).

On peut y voir également le signe de la capacité des «machines à communiquer», que sont les entreprises, à produire des discours mythiques, soit des discours totalisants, idéologiques et incantatoires, perpétuant les grands mythes de l'humanité : «conjuration de la mort, connaissance exhaustive, ubiquité, progrès et foi » (Perriault, 1989, p. 74-75). Cette persistance d'une «pensée mythique» face à une pensée techno-stratégique, bien décrite par Jacques Perriault, s'inscrit dans un héritage de pensée des mythes associés aux techniques, dans leur dimension sémiotique, mais également historique, sociocognitive, sociologique et philosophique.

Or, ce récit utopique d'un progrès universel généré par la technologie numérique qui renvoie en droite ligne aux origines du média Internet, semble quelque peu battu en brèche par la professionnalisation de nouvelles fonctions du numérique comme celle d'évangéliste.

En effet, à ce dernier, nous l'avons vu, est associé un ethos d'expertise, lequel semble entrer en contradiction avec le principe démocratique affiché et la «présupposition d'égalité »(Cardon, 2010, p. 78) des acteurs intervenant sur Internet ou tout autre support numérique. La communication, devenue digitale, reste assurée par une minorité de professionnels sous l'égide de la direction de l'entreprise. Alors même que les discours véhiculent l'idée d'un outil capable d'aplanir toute différence en termes de compétences techniques et d'interactions s'établissant sur une base formelle d'égalité, la hiérarchisation n'en est toutefois pas absente; elle participe de la construction d'un ethos professionnel et en sert de caution.

\section{Conclusion}

«L'irruption d'une nouvelle technologie s'accompagne toujours d'une floraison de discours, cherchant à prédire quelles pourraient en être les conséquences sur nos modes de vie. Internet n'échappe pas à cette règle, bien au contraire " (Rebillard, 2007 , p. 83). Nous avons fait le choix d'aborder la question de l'émergence d'une nouvelle fonction de communicateur liée à l'essor de la technologie numérique sous l'angle de l'analyse des discours d'escorte auxquels elle donne lieu.

La prégnance du registre religieux, et notamment la rhétorique messianique que nous avons pu mettre au jour, interroge les imaginaires de la communication sousjacents à travers une conception de la technique comme enchantement. Dans cette perspective, faut-il voir dans le triple récit élaboré et véhiculé autour de la technologie numérique - récit de la conquête, de l'émancipation et de la 
communauté - une "réponse à la crise des "grands" récits d'émancipation de 1'humanité » (D'Almeida, 2004, p. 27) ${ }^{62}$ ?

Il apparaît en tout cas que le récit utopique du progrès, à destination universelle et démocratique, permis par le numérique, se heurte dans les discours des deux entreprises étudiées à des logiques organisationnelles et professionnelles. L'exploration des logiques d'ethos entourant le métier d'évangéliste révèle que le recours à la catégorie de l'expertise, à des fins de légitimation professionnelle, entre en dissonance avec la «présupposition d'égalité » attachée à Internet. On voit alors, comme le soulignent Proulx et Millerand, que « la capacité à élaborer des contenus particuliers et la compétence à communiquer dans les environnements numériques ne sauraient se passer d'un travail proprement politique de mise en visibilité, de reconnaissance et de légitimation » (Proulx et Millerand, 2010, p. 20).

Notre approche, centrée sur l'analyse des médiations langagières et discursives, pourrait donc être utilement complétée par une analyse en termes sociologiques, empruntant notamment à la sociologie des professions, afin de préciser la caractérisation de ce nouveau métier du numérique sur lequel très peu de données statistiques sont à ce jour disponibles ${ }^{63}$, de même qu'une approche en termes culturels, voire interculturels : on songe ici aux questions relatives à l'origine nordaméricaine du terme d'evangelist - et à ce qu'elle révèle plus largement quant à l'adoption de termes anglo-saxons par les entreprises françaises, au rapport étroit entre technologie et religion dans ce contexte culturel spécifique, tel qu'il se fait jour notamment à travers l'idée de messianisme technologique.

\section{Bibliographie}

Adam, J-M. (2001). Les textes : types et prototypes. Paris : Nathan Université.

Adam, J-M. (2002). Récit. Dans P. Charaudeau et D. Maingueneau (dir.). Dictionnaire d'analyse du discours (p. 484-487). Paris : Éditions du Seuil.

Amossy, R. (2010). La présentation de soi. Ethos et identité verbale. Paris : Presses Universitaires de France.

Austin, J. L. (1970). Quand dire, c'est faire. Paris : Editions du Seuil.

\footnotetext{
${ }^{62}$ Rappelons que Jean-François Lyotard voyait dans «l'incrédulité à l'égard des méta-récits 》 une caractéristique de la postmodernité (Lyotard, 1979, p. 7).

${ }^{63}$ C'est particulièrement le cas en France, contrairement au contexte anglo-saxon : le « Portail des métiers de l'Internet» du Ministère français de l'économie, de l'industrie et du numérique ne fait par exemple pas mention de la fonction d'évangéliste technologique : http://metiers.internet.gouv.fr
} 
68 Le communicateur bousculé par le numérique

Baumgartner, E. et Ménard, P. (1996). Dictionnaire étymologique et historique de la langue française. Paris : Librairie Générale Française.

Bautier, R. (1994). De la rhétorique à la communication. Grenoble: Presses Universitaires de Grenoble.

Bonaccorsi, J. (2013). Approches sémiologiques du web. Dans C. Barats (dir.). Manuel d'analyse du web (p. 125-146). Paris : Armand Colin.

Bonnafous, S. et Krieg-Planque, A. (2013). L'analyse du discours. In S. Olivesi (dir.). Sciences de l'information et de la communication. Objets, savoirs, discipline (p. 223-238). Grenoble : Presses Universitaires de Grenoble.

Breton, P. (2000). Le culte de l'Internet. Une menace pour le lien social ? Paris : La Découverte.

Cardon, D. (2010). La démocratie Internet. Promesses et limites. Paris : Éditions du Seuil.

D’Almeida, N. (2001). Les promesses de la communication. Paris: Presses Universitaires de France.

D’Almeida, N. (2004). Les organisations entre récits et médias. Canadian Journal of Communication, 29, 25-46.

Danblon, E. (2005). La fonction persuasive. Anthropologie du discours rhétorique : origines et actualité. Paris : Armand Colin.

De la Broise, P. et Brulois, V. (2010). La communication interne aux prises avec la professionnalisation. Revue internationale de communication sociale et publique, (3-4), 123-134.

Doueihi, M. (2011). La grande conversion numérique. Paris : Éditions du Seuil.

Douyère, D. (2010a). La communication sociale: une perspective de l'Eglise catholique ? Jean Devèze et la critique de la notion de "communication sociale", Revue internationale de communication sociale et publique, (3-4), 73-86.

Douyère, D. (2010b). Une organisation fondée pour communiquer : 1'Ordre des Frères prêcheurs (1215-1228). Dans C., Loneux et B., Parent. Communication des organisations: recherches récentes, Tome 1 (p. 145-152). Paris : L'Harmattan.

Ferrary, M. et Pesqueux, Y. (2011). Management de la connaissance, Paris : Economica.

Foucault, M. (1969). L'archéologie du savoir. Paris : Gallimard. 
Giroux, N. et Marroquin, L. (2005). L'approche narrative des organisations, Revue française de gestion, 31(159), 15-42.

Jakobson, R. (1963). Essais de linguistique générale. Paris : Editions de Minuit.

Kerbrat-Orecchioni, C. (2002). Acte de langage. Dans P., Charaudeau et D. Maingueneau (2002). Dictionnaire d'analyse du discours (p. 16-19). Paris : Editions du Seuil.

Krieg-Planque, A. et Oger, C. (2010). Discours institutionnels : perspectives pour les sciences de la communication. Mots. Les langages du politique. Lyon, ENS Editions, (94), 91-96.

Krieg-Planque, A. (2012). Analyser les discours institutionnels. Paris: Armand Colin.

Latour, B. (2010). Prendre le pli des techniques. Réseaux, (163), 20-31.

Lyotard, J-F. (1979). La condition postmoderne. Paris : Editions de Minuit.

Mila, D. (2013). La confiance et les modes de management comme facteurs explicatifs $d u$ partage des connaissances digitales dans un contexte d'apparition d'une nouvelle génération. Mémoire de Master 2 «Communication des entreprises », Université Paris 13, 123 p.

Monnoyer-Smith, L. (2013). Le web comme dispositif: comment appréhender le complexe? Dans C. Barats (dir.). Manuel d'analyse du web. (p. 12-31). Paris : Armand Colin.

Perriault, J. (1989). La logique de l'usage. Essai sur les machines à communiquer. Paris : Flammarion.

Proulx, S. et Millerand, F. (2010). Le Web social, au carrefour de multiples questionnements. Dans F. Millerand, S. Proulx et J. Rueff (dir.). Web social. Mutation de la communication. (p. 13-30). Québec : Presses de 1'Université du Québec.

Rebillard, F. (2007). Le Web 2.0 en perspective. Une analyse socio-économique de l'internet. Paris : L'Harmattan.

Todorov, T. (1978). Les genres du discours. Paris : Editions du Seuil.

Weber, M. (2000). L'éthique protestante ou l'esprit du capitalisme. Paris: Flammarion. 